\title{
Neuropatía del pudendo como causa de dolor pélvico
}

\section{Pudendal neuropathy as a cause of pelvic pain}

\author{
Ana María Ortiz Zableh, MD ${ }^{4}$ \\ 1 Universidad Autónoma de Bucaramanga; Uróloga Pontificia \\ Universidad Javeriana; Máster Universitario de Investigación Clínica \\ en Donación y Trasplante de Órganos, Tejidos y Células, Universidad \\ de Barcelona; Uróloga, Centro Urológico Foscal, Floridablanca, \\ Santander, Colombia \\ 2 Universidad Industrial de Santander; Anestesióloga, Universidad \\ Militar Nueva Granada; Fellow Anestesia Regional guiada por \\ Ultrasonido programa CLASA-WFSA-SBA; Anestesióloga Sociedad \\ Especializada de Anestesiología SEA S.A., Clínica Carlos Ardila Lulle, \\ Floridablanca, Santander, Colombia \\ 3 Universidad Autónoma de Bucaramanga; Residente de Segundo año \\ de Urología, Universidad Autónoma de Bucaramanga, Floridablanca, \\ Santander, Colombia \\ ${ }^{4}$ Interna, Pontificia Universidad Javeriana, Bogotá, Colombia
}

Verónica Tobar Roa, MD ${ }^{1}$ María Fernanda Rojas Gómez, MD² Ana María Gómez González, MD³

\begin{abstract}
Address for correspondence María Fernanda Rojas Gómez, MD, Universidad Industrial de Santander; Anestesióloga, Universidad Militar Nueva Granada; Fellow Anestesia Regional guiada por Ultrasonido programa CLASA-WFSA-SBA; Anestesióloga Sociedad Especializada de Anestesiología SEA S.A., Clínica Carlos Ardila Lulle, Floridablanca, Santander, Colombia (e-mail: mariafernanda.mfrojas@gmail.com).
\end{abstract}

Urol Colomb 2018;27:25-34.
Resumen
Palabras clave
- nervio pudendo
- neuralgia
- atrapamiento nervioso
- dolor crónico
- canal de Alcock
- dolor pélvico

El síndrome de atrapamiento del nervio pudendo es una de múltiples causas de dolor pélvico crónico. Hemos realizado una revisión de la literatura sobre su presentación clínica, diagnóstico y tratamiento, con el propósito de conocer los detalles más relevantes de una enfermedad que cada vez está siendo más diagnosticada, con el fin de realizar un abordaje precoz desde un punto de vista integral.

Pudendal neuralgia due to pudendal nerve entrapment is one of the many causes of chronic pelvic pain. A literature was carried out as regards its clinical presentation, and diagnostic and therapeutic approach, in order to understand the most relevant details of this disorder that is increasingly being diagnosed, with the purpose of implementing an early approach from an integral perspective. received

February 21, 2017

accepted

April 16, 2017

published online

January 10, 2018
DOI https://doi.org/

10.1016/j.uroco.2017.04.005.

ISSN 0120-789X.

eISSN 2027-0119.
Copyright $\odot$ 2018, Sociedad Colombiana License terms de Urología. Publicado por Thieme Revinter Publicações Ltda., Rio de Janeiro, Brazil. Todos los derechos reservados. 


\section{Introducción}

El síndrome de atrapamiento del nervio pudendo fue descrito por primera vez en 1987 por el neurólogo francés Gerard Amarenco, refiriéndose al dolor perineal que presentaban algunos ciclistas y que en estudios de electrofisiología se correlacionaba con el atrapamiento del nervio a su paso por el canal de Alcock; fue denominado inicialmente "parálisis perineal del ciclista»., ${ }^{1,2}$

El síndrome se caracteriza por un dolor neuropático en el trayecto del nervio pudendo, ${ }^{3-5}$ que empeora en el curso del día o con la posición sentada sin déficit sensitivo asociado. Es rara vez sospechado, identificado y diagnosticado, ya que sus síntomas diversos pueden simular otras enfermedades, incluyendo trastornos psiquiátricos. ${ }^{6}$ La incidencia de esta enfermedad es de $1 / 100.000$ en la población general ${ }^{7}-$ siendo el género femenino el más afectado-. De acuerdo con Orphanet, la neuralgia del pudendo está presente en un $4 \%$ de los pacientes que consultan por dolor, afectando a 7 mujeres por cada 3 hombres; ${ }^{8}$ sin embargo se considera que su incidencia y prevalencia están subestimadas. ${ }^{7,9}$

El objetivo del presente artículo es realizar una revisión de los aspectos más relevantes de la neuropatía del nervio pudendo, con el fin de dar a conocer y fomentar un mejor abordaje diagnóstico y terapéutico de esta enfermedad, aún desconocida para muchos.

Estrategia de búsqueda: Se realizó una búsqueda de la literatura a través de Medline, Embase y Science Direct, desde febrero de 1985 hasta febrero de 2017, evaluando los artículos relevantes con respecto a la neuropatía del pudendo, utilizando las palabras clave: anatomía del nervio pudendo, nervio pudendo, neuralgia del pudendo, atrapamiento nervioso, dolor crónico, canal de Alcock y dolor pélvico. La búsqueda se limitó a artículos publicados en español, inglés y francés.

\section{Anatomía del nervio pudendo}

El nervio pudendo ha mostrado gran variabilidad anatómica., ${ }^{40-16}$ Recibe aportes principalmente de las raíces ventrales S2-S4 del plexo sacro, con contribuciones variables de S1 y S5. ${ }^{17,18}$ Es un nervio mixto por el cual discurren fibras motoras, sensitivas y autonómicas. Se han descrito 3 ramificaciones del nervio: la rama rectal inferior, la rama perineal y la dorsal del pene/clítoris. La rama perineal se divide en superficial y profunda, la rama superficial se divide en medial y posterolateral del escroto/labio. ${ }^{5}$ Son frecuentes las interconexiones entre la rama perineal, la rectal y el nervio femorocutáneo posterior. ${ }^{14}$ Es de destacar la importancia funcional en la micción, defecación, erección, eyaculación y parto. ${ }^{11,14,19-21}$

El nervio pudendo emerge de la pelvis a través del foramen ciático mayor, con un trayecto caudal al músculo piriforme y al nervio ciático. Posteriormente reingresa a la pelvis a través del agujero ciático menor, dorsal al ligamento sacroespinoso y ventral al ligamento sacrotuberoso ${ }^{10,13,22}$ como se muestra en la - Figura 1. En este trayecto interligamentoso es donde frecuentemente podemos

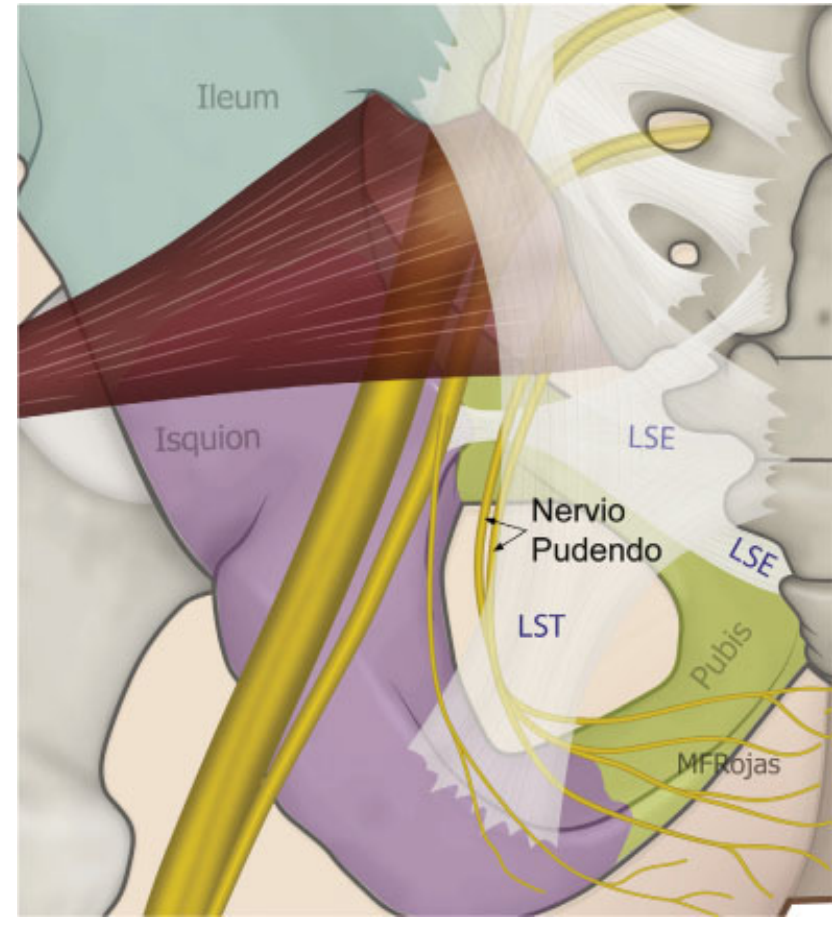

Fig. 1 Esquema del trayecto del nervio pudendo en el área glútea. LSE: ligamento sacroespinoso; LST: ligamento sacrotuberoso.

encontrar enfermedades compresivas del nervio. ${ }^{16}$ Finalmente el nervio continúa por el repliegue del músculo obturador interno o canal de Alcock hacia la región perineal. $^{10,16}$

En las descripciones anatómicas realizadas por los Dres. Gabrielli y Olave ${ }^{14}$ se vio que el nervio puede presentarse como tronco único (53,3\%), como 2 troncos (36,7\%) y como 3 troncos (6,7\%). Mahakkanukrauh et al. ${ }^{13}$ encontraron el nervio pudendo como un tronco (56,2\%), 2 troncos (31,5\%) y 3 troncos (12,3\%). Furtmüller et al. ${ }^{15}$ en sus evaluaciones anatómicas encontraron que en la mayoría de las veces la rama perineal y la rama rectal provienen en un tronco común; también hallaron tronco común entre la rama dorsal del pene y la perineal; menos frecuentemente entre la dorsal del pene y la rectal inferior, formando de esta manera 2 y hasta 3 troncos desde su origen.

En la parte posterior de la fosa isquiorrectal, inmediatamente después de pasar el ligamento sacroespinoso, el nervio da su primera rama: el nervio rectal inferior, que penetra la fascia interna del músculo obturador para distribuirse en su territorio anal. ${ }^{17,23}$ Mahakkanukrauh et al. encontraron que en el $21 \%$ de las veces el nervio rectal inferior se origina independientemente de la raíz de S4 sin hacer parte del tronco del nervio pudendo. ${ }^{13}$ La rama perineal penetra medialmente la fascia interna del músculo obturador deslizándose hacia la base del diafragma urogenital donde se bifurca en sus ramas terminales superficial y profunda. La rama dorsal del pene/ clítoris sale del canal de Alcock ${ }^{11}$ y continúa próxima la rama púbica inferior del hueso púbico hacia la región anterior; el trayecto del nervio en el canal de Alcock es una de las zonas 
descritas más susceptible de presentar atrapamiento después del trayecto interligamentario. ${ }^{16}$ O'Bichere et al. mostraron en sus trabajos que la rama dorsal del pene/ clítoris, puede originarse directamente de S2 en un $25 \%$ de las veces. $^{24}$

El 30\% del nervio está constituido por fibras autonómicas que intervienen en la erección y en la sensación de distención vesical y rectal. ${ }^{25,26} \mathrm{El} 70 \%$ son fibras somáticas, de las cuales el $50 \%$ son sensitivas e inervan la piel del periné y de los genitales externos; y el 20\% son motoras e inervan el esfínter anal externo, el músculo elevador del ano, el músculo bulbo e isquiocavernoso, el esfínter estriado de la uretra y los músculos perineales profundos y superficiales. ${ }^{27-29}$ Detalles anatómicos adicionales se presentan en la -Figura 2.

\section{Fisiopatología}

La lesión del nervio puede presentarse por procesos mecánicos, infecciosos 0 inmunológicos. ${ }^{16,30}$ La compresión mecánica frecuentemente es secundaria a atrapamiento del nervio o a causas neurovasculares que incluyen varices o múltiples interconexiones venosas; ${ }^{31}$ sin embargo existen otras causas como espasmo muscular del piso pélvico, o tejido cicatricial por cirugías previas. ${ }^{3} \mathrm{El}$ mecanismo de lesión puede ser producido principalmente por compresión o estiramiento de la fibra nerviosa. ${ }^{32}$ Dichas lesiones generan compromiso de los vasa nervorum, alteración de la permeabilidad vascular, proliferación de fibroblastos, edema local o trastornos iónicos que comprometen la conducción nerviosa. La persistencia de la lesión puede terminar en desmielinización progresiva del nervio, alteración de la fosforilación oxidativa y producción de ATP, ocasionando una alteración de la bomba de $\mathrm{Na}-\mathrm{K}$, el transporte axoplásmico y la integridad de la membrana, que comprometen la fisiología normal de la fibra. ${ }^{33}$ La profundidad y reversibilidad del daño dependerá del tiempo de evolución de la lesión.

La causa del síndrome no siempre es clara. Sin embargo, al realizar un interrogatorio exhaustivo, es posible encontrar un antecedente de compresión o estiramiento del nervio; por ejemplo, haber practicado ciclismo, equitación, permanecer sentado por periodos prolongados de tiempo, antecedente

\begin{tabular}{|c|c|c|c|c|c|c|}
\hline Raices Nerviosas & \multicolumn{3}{|c|}{ Ramificaciones } & Inervación sensitiva & Inervación motora & Vegetativo \\
\hline \multirow{2}{*}{$\begin{array}{c}\text { Nervio pudendo } \\
\text { (Np) } \\
\text { S1 S2-S4 S5 } \\
\text { Presentación } \\
\text { anatómica } \\
\text { en 1, } 203 \text { troncos }\end{array}$} & \multicolumn{2}{|c|}{$\begin{array}{l}\text { Rama Dorsal del } \\
\text { pene /clitoris S2 } \\
(\mathrm{RDp} / \mathrm{c})\end{array}$} & $\begin{array}{l}\text { Rama clitoridea } \\
\text { Rama pubiana }\end{array}$ & $\begin{array}{l}\text { Tejido eréctil del cuerpo cavernoso } \\
\text { y cruz del pene/clitoris y la piel que } \\
\text { cubre el aspecto dorsolateral del } \\
\text { prepucio, glande y pene / clitoris }\end{array}$ & $\begin{array}{l}\text { Músculos involucrados en la } \\
\text { erección }\end{array}$ & $\begin{array}{l}\text { Iniciación y } \\
\text { mantenimiento } \\
\text { de la erección } \\
\text { (parasimpático) }\end{array}$ \\
\hline & \multirow[t]{2}{*}{$\begin{array}{l}\text { Rama } \\
\text { Perineal } \\
\text { (Rp) }\end{array}$} & \multicolumn{2}{|c|}{ Profunda / muscular (p) } & $\begin{array}{l}\text { Tercio inferior de la vagina y de la } \\
\text { uretra }\end{array}$ & $\begin{array}{l}\text { Músculo perineal transverso superfi- } \\
\text { cial y profundo, bulboesponjoso, } \\
\text { isquiocavernoso esfinter ureteral, } \\
\text { porción ant del esfinter anal ext. y } \\
\text { areas variables del músculo } \\
\text { elevador del ano }\end{array}$ & $\begin{array}{l}\text { Sensación } \\
\text { consciente de } \\
\text { necesidad } \\
\text { urinaria }\end{array}$ \\
\hline \multirow{2}{*}{$\begin{array}{l}30 \% \text { autonómico y } \\
70 \% \text { somática: } 50 \% \\
\text { sensitivo y } 20 \% \\
\text { motor }\end{array}$} & & $\begin{array}{l}\text { Superficial } \\
\text { (s) }\end{array}$ & \begin{tabular}{|l|} 
Rama medial \\
Rama \\
posterolateral \\
\end{tabular} & $\begin{array}{l}\text { Porción posterior de la piel del } \\
\text { perine y escroto/labios mayores y } \\
\text { menores }\end{array}$ & $\begin{array}{l}\text { Al esfinter externo e inervación } \\
\text { variable del músculo elevador del } \\
\text { ano }\end{array}$ & \\
\hline & \multicolumn{3}{|c|}{ Rama rectal inferior S3 (Rri) } & $\begin{array}{l}\text { Inervación sensitiva de la piel de la } \\
\text { circunferencia anal, el tercio } \\
\text { caudal del recto y posterior vulvar }\end{array}$ & $\begin{array}{l}\text { Músculo elevador del ano y del } \\
\text { esfinter externo del ano }\end{array}$ & $\begin{array}{l}\text { Sensación cons- } \\
\text { ciente de necesi- } \\
\text { dad de defecar }\end{array}$ \\
\hline
\end{tabular}

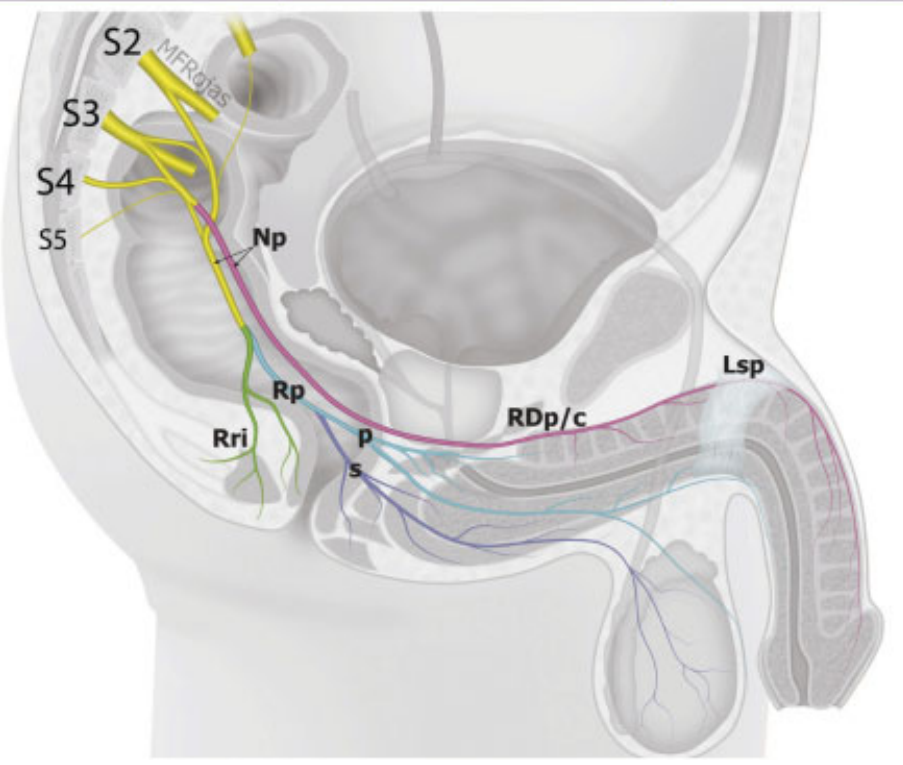

Fig. 2 Anatomía del nervio pudendo (Np). Lsp: ligamento suspensorio del pene; p: profunda; RDp/c: rama dorsal del pene/clítoris; Rp: rama perineal; Rri: rama rectal inferior; s: superficial. 
de hematomas pélvicos, partos vaginales, radioterapia de órganos pélvicos que provoque cambios morfológicos o estructurales en los tejidos adyacentes ${ }^{34,35}$ o cirugías pélvicas y ortopédicas, entre otros. ${ }^{1,15,25,33}$

Dentro de las cirugías más relacionadas con el síndrome se encuentran la rectopexia y la sacrocolpopexia laparoscópica. ${ }^{18,26,36}$ Sin embargo, cualquier intervención quirúrgica en la zona pélvica puede inducir el atrapamiento o la formación de neuromas de este o de otros nervios locales, ${ }^{29}$ como en la cirugía de corrección de incontinencia urinaria con cinta libre de tensión. ${ }^{37}$ Chung et al. encontraron neuropatía del pudendo en el 1,6\% de las pacientes llevadas a cirugía de suspensión del ligamento uterosacro. ${ }^{38}$ Bondar et al. también reportaron una frecuencia del $15 \%$ en pacientes llevados a osteosíntesis de fracturas de fémur en mesa de fracturas con soporte pélvico. ${ }^{25}$

\section{Clínica}

En las mujeres el dolor se localiza a nivel de la vulva, vagina, clítoris, periné y recto; mientras que en los hombres se localiza en el glande, escroto, periné y recto. La presentación clásica es unilateral, aunque se han reportado algunos casos con afectación bilateral. ${ }^{3}$ El dolor puede ser paroxístico o constante, e independiente del estímulo. Se describe como una descarga, lancinante o quemante y se asocia a parestesias, disestesias, alodinia, híper- o hipoalgesia. De forma característica se exacerba al sentarse sobre una superficie plana y se alivia en decúbito; en la mañana los síntomas son escasos y aumentan durante el día. Algunas pacientes refieren dispareunia ocasional asociada a anorgasmia; en los hombres, puede haber dolor durante la erección, dificultad para mantenerla o dolor en la eyaculación. También pueden presentarse alteraciones durante la micción o la defecación. ${ }^{39}$

El dolor suele llevar a algunos pacientes a adoptar una posición antiálgica, sentándose solo sobre la hemipelvis sana. ${ }^{3,9}$

Hay 6 tipos de presentación del síndrome: ${ }^{2} 1$. Solo dolor. 2 . Dolor asociado a síntomas urinarios del tracto de salida. 3 . Dolor asociado a dificultad para la defecación. 4. Dolor con disfunción sexual. 5. Dolor con incontinencia urinaria, fecal o ambas. 6. Combinaciones.

\section{Examen físico}

Con el paciente en posición de litotomía se busca el signo de Tinel, que es la reproducción del dolor al palpar la espina isquiática, ya sea por vía vaginal o rectal. ${ }^{6,34,39}$ También es útil el test de la pinza rodada (rolling test), que consiste en pinzar con los dedos la piel y el tejido celular subcutáneo, desplazándolo desde el ano hacia el pubis sobre el trayecto de las ramas del nervio pudendo, reproduciendo los síntomas dolorosos como se muestra en la - Figura 3. En los dermatomas comprometidos (-Fig. 4), es frecuente encontrar alteraciones de la sensibilidad. ${ }^{34,39}$

Se han encontrado cambios cutáneos como resultado del compromiso de las fibras autonómicas, incluyendo: piel de naranja, livedo reticularis, piel anserina y eritema en labios. ${ }^{9,40}$

\section{Diagnóstico}

El diagnóstico es principalmente clínico. Está basado en los criterios de Nantes ${ }^{6,33}$ mostrados en la - Tabla 1, los cuales se complementan con los criterios de Benson y Griffis ${ }^{5}$ (-Tabla 2). En el diagnóstico, también se plantean criterios mayores y menores, $, 9,25,33,39$ acorde a lo listado en la - Tabla 3.

Cuando el paciente tiene síntomas atípicos o no cumple los criterios diagnósticos mencionados, se deben descartar otras enfermedades como compresión extrínseca por tumores o neuromas, o compromiso de las raíces del plexo sacro. $^{41}$

\section{Pruebas diagnósticas}

La utilidad de la electrofisiología en esta enfermedad es limitada, con baja sensibilidad y especificidad. Caracteriza las alteraciones en la conducción nerviosa, sin identificar la causa o el sitio anatómico puntual de la lesión. ${ }^{42}$ Entre las pruebas que se han utilizado, las que han demostrado mayor
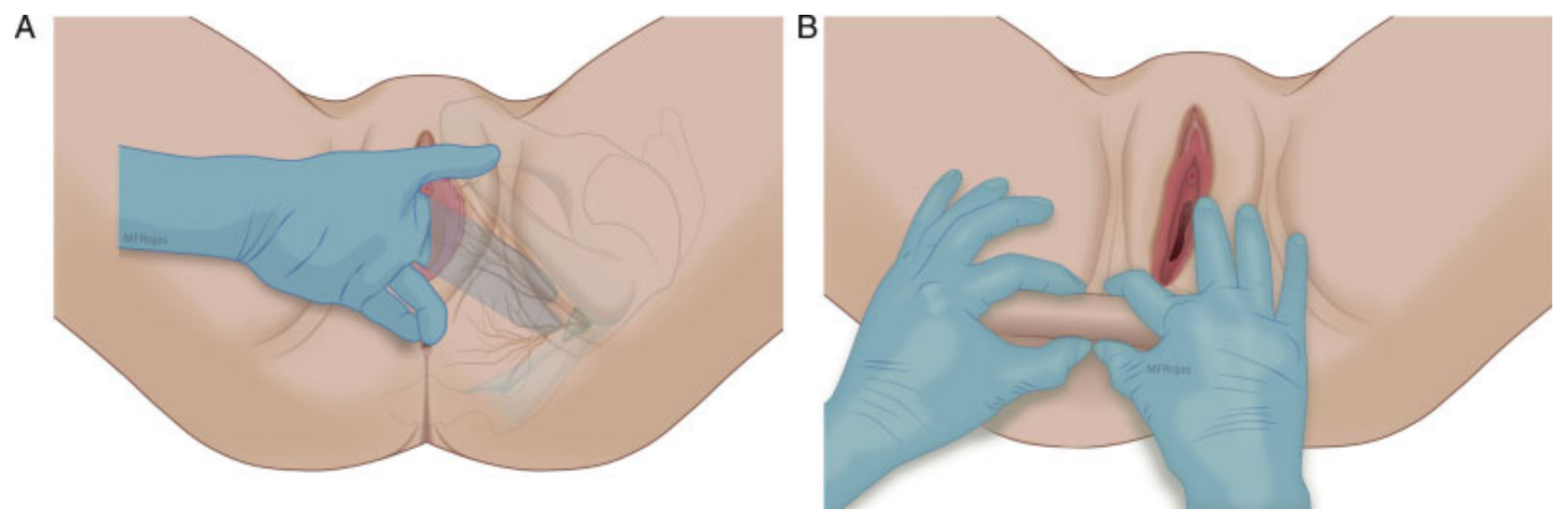

Fig. 3 A: Signo de Tinel. B: Test de la pinza rodada. 


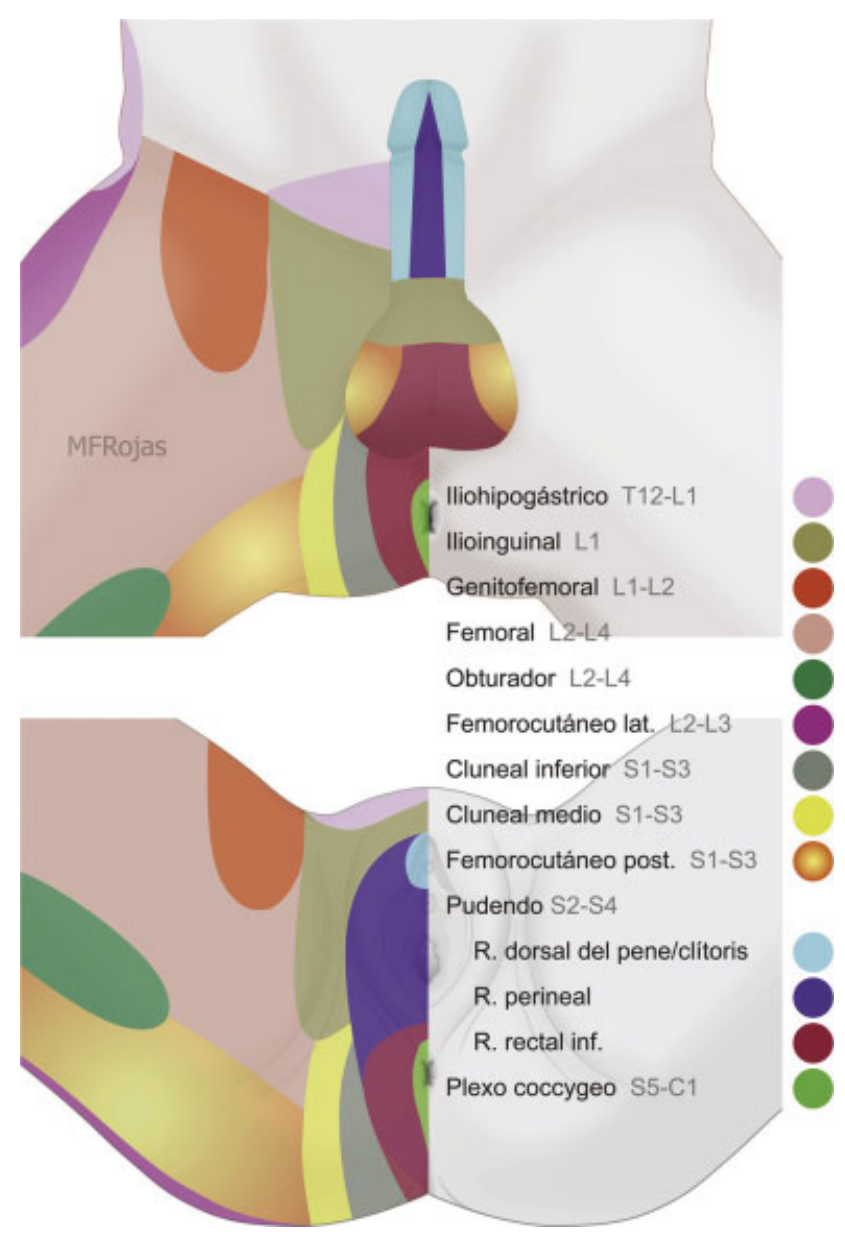

Fig. 4 Dermatomas de la región perineal y muslo proximal.

utilidad son la electromiografía con aguja y los estudios de conducción nerviosa. ${ }^{43}$

La electromiografía se realiza en el esfínter anal externo y los músculos bulbocavernosos, haciendo una comparación entre ambos lados.

La prueba de latencia motora terminal del nervio pudendo evalúa la conducción nerviosa, realizando un estímulo transrectal a nivel de las espinas isquiáticas; si la latencia es mayor a 2,2 ms, es posible que haya lesión del nervio. ${ }^{43-45}$

El test de latencia del reflejo bulbocavernoso es útil para explorar las vías aferentes del arco reflejo sacro, pero la respuesta puede ser retardada en lesiones motoras eferentes puras. ${ }^{46}$ Otros estudios incluyen los umbrales sensoriales, que se realizan mediante la estimulación eléctrica sobre el nervio dorsal del pene/clítoris, identificando el umbral de percepción y dolor. Los potenciales evocados sensoriales, que evalúan la vía sensitiva periférica y medular, miden el tiempo que tarda un estímulo eléctrico en recorrer el nervio periférico hasta la corteza parietal, siendo el valor normal de este tiempo de $40 \mathrm{~ms}^{2}$.

Más recientemente se ha descrito la utilidad del doppler color de la arteria pudenda, que ha mostrado resultados prometedores en el proceso diagnóstico del síndrome en mención. El procedimiento se realiza midiendo las velocidades de flujo e índices de resistencia de la arteria pudenda antes y después de la pinza interligamentaria y el canal de Alcock. ${ }^{42}$

En los pacientes con disfunción miccional y síntomas urinarios irritativos es recomendable realizar un estudio urodinámico para descartar otras enfermedades.

\section{Imágenes diagnósticas}

La radiografía, la resonancia magnética de pelvis y la gammagrafía ósea son útiles para descartar otras enfermedades, como entidades neurológicas, neoplásicas y ortopédicas, entre otras. ${ }^{4,39,42,47}$

Dentro del estudio de resonancia magnética se pueden realizar diferentes técnicas. Las 3 principales son la neurografía de difusión, la tractografía con tensor de difusión y la neurografía basada en T2. La tractografía periférica es la más utilizada y evalúa el curso, calibre, patrón fascicular, tamaño y fibrosis perineural, así como la presencia de lesiones o masas. ${ }^{48}$ En el caso de neuropatía del pudendo, se puede observar hiperintensidad del haz neurovascular, edema y asimetría a lo largo de la cara medial del músculo obturador interno y a su entrada en el canal de Alcock. ${ }^{49}$ Otros hallazgos pueden ser distorsiones del curso del nervio o contornos anormales, que pueden estar en relación con atrapamiento o adherencias, así como efectos postraumáticos. ${ }^{50,51}$

\section{Diagnóstico diferencial}

Se deben descartar otras enfermedades como tumores, endometriosis, neuromas, miomatosis uterina o várices pélvicas que puedan causar síntomas similares al comprimir el nervio; ${ }^{51,52}$ u otras enfermedades como el síndrome de vejiga dolorosa, la vulvodinia, el síndrome piriforme, el síndrome de cauda equina, atrapamiento de otros nervios como el obturador, el genitofemoral, el cluneal inferior o el ilioinguinal-. ${ }^{43,53}$ También presentan una sintomatología similar la prostatitis crónica y el síndrome de dolor miofascial del piso pélvico., ${ }^{3,2833,43}$ En pacientes femeninas cuya principal queja es la alteración de la micción, se debe considerar la posibilidad de un síndrome de Fowler. ${ }^{54}$

\section{Tratamiento}

El abordaje terapéutico debe asumirse de manera temprana pero secuencial de acuerdo a la etiología, empezando siempre por el manejo conservador. Se recomienda implementar medidas básicas para aminorar los síntomas: suspender las actividades que desencadenan el dolor, evitar periodos prolongados en posición sentada o utilizar plataformas perineales para apoyar el peso corporal sobre las tuberosidades isquiáticas, dejando el periné libre de presión. 6,8

La fisioterapia es, para muchos autores, la primera línea de tratamiento. $^{3}$ El fisioterapeuta debe enfocarse en la búsqueda de áreas de hipertonía en la musculatura del piso pélvico. ${ }^{55}$ Existen múltiples técnicas para la liberación de espasmos, educación postural y ejercicios de estiramiento. ${ }^{7,56}$ También son útiles la estimulación eléctrica y el biofeedback. Rhame et al. reportaron el caso 
Tabla 1 Criterios de Nantes para el diagnóstico de neuralgia del nervio pudendo

\begin{tabular}{|c|c|}
\hline \multirow{5}{*}{$\begin{array}{l}\text { Criterios } \\
\text { esenciales del } \\
\text { diagnóstico } \\
\text { (todos deben } \\
\text { estar presentes) }\end{array}$} & Dolor en el territorio del nervio pudendo \\
\hline & El dolor se incrementa al sentarse en una superficie plana \\
\hline & El dolor no despierta al paciente durante la noche \\
\hline & Dolor sin deterioro sensorial predominante \\
\hline & Alivio mediante bloqueo anestésico del nervio pudendo \\
\hline \multirow{8}{*}{$\begin{array}{l}\text { Criterios } \\
\text { complementarios } \\
\text { del diagnóstico }\end{array}$} & Dolor de tipo neuropático \\
\hline & Alodinia o hiperpatía \\
\hline & Sensación de cuerpo extraño rectal o vaginal \\
\hline & Incremento del dolor durante el día \\
\hline & Dolor predominantemente unilateral \\
\hline & Dolor desencadenado por la defecación \\
\hline & Presencia de dolor a la palpación de la espina isquiática \\
\hline & Hallazgos neurofisiológicos positivos en hombres o en mujeres nulíparas \\
\hline \multirow{7}{*}{$\begin{array}{l}\text { Signos asociados } \\
\text { sin excluir el } \\
\text { diagnóstico }\end{array}$} & Dolor en la nalga al sentarse \\
\hline & Dolor referido a la cara medial del muslo \\
\hline & Alteración de la frecuencia urinaria y/o dolor cuando hay vejiga llena \\
\hline & Dolor que se produce después de la eyaculación, dispareunia y/o dolor después del coito \\
\hline & Dolor suprapúbico \\
\hline & Exploración neurofisiológica normal \\
\hline & Disfunción eréctil \\
\hline \multirow[t]{4}{*}{$\begin{array}{l}\text { Criterios de } \\
\text { exclusión }\end{array}$} & $\begin{array}{l}\text { Dolor en glúteos, pubis, coccígeo o en hipogastrio que no corresponde con el territorio anatómico } \\
\text { del nervio pudendo }\end{array}$ \\
\hline & $\begin{array}{l}\text { Prurito: es principalmente sugestivo de una lesión dermatológica (atrófica, liquen plano, etc.) en } \\
\text { lugar de una lesión nerviosa }\end{array}$ \\
\hline & $\begin{array}{l}\text { Dolor exclusivamente paroxístico: es sugestivo de una lesión compresiva, justifica una investigación } \\
\text { adicional con imágenes para descartar neurofibroma o schwannoma, enfermedad de la cola de } \\
\text { caballo (schwannoma sacro) y la médula espinal (meningioma) }\end{array}$ \\
\hline & Pruebas de imágenes anormales que expliquen otras causas de dolor \\
\hline
\end{tabular}

de una paciente con 2 años de neuralgia severa del pudendo refractaria a múltiples tratamientos, quien presentó mejoría significativa y de larga duración con radiofrecuencia. ${ }^{57}$

Dentro del arsenal terapéutico se encuentran los relajantes musculares y los neuromoduladores (como gabapentina, pregabalina, ciclobenzaprina), analgésicos antiinflamatorios, antidepresivos tricíclicos, lidocaína en gel o en parches y opioides. ${ }^{4,6,9}$ Pereira et al. demostraron en su estudio que el $42 \%$ de los pacientes con neuralgia del pudendo respondieron al tratamiento de primera línea con lidocaína tópica al $5 \%$ combinada con amitriptilina $10 \mathrm{mg}$ vía oral/12 h durante 6-8 semanas, y que de los pacientes que no respondieron a este manejo inicial, el 31\% mejoró sus síntomas cuando se adicionó tramadol. ${ }^{51}$

También se ha reportado utilidad de la toxina botulínica para reducir la contracción del músculo obturador. ${ }^{9,50,58-60}$ Recientemente se ha descrito mejoría en la sintomatología con el uso de parches dérmicos de capsaicina que actúa sobre receptores nocioceptivos modulando el movimiento iónico a través de la membrana; como una alternativa terapéutica con resultados moderados y que deben seguir siendo evaluados. ${ }^{61}$ Calabrò et al. reportaron un caso exitoso de tratamiento médico con palmitoiletanolamida, un lípido con efecto antiinflamatorio y antinocioceptivo. ${ }^{62}$

\section{Bloqueo anestésico del nervio pudendo}

El bloqueo del nervio pudendo se utiliza como prueba diagnóstica y terapéutica. Puede ser realizado a través de múltiples abordajes - transperineal, transglúteo, transrectal y transvaginal-. En los abordajes proximales o posteriores, la espina isquiática es el referente anatómico más importante. ${ }^{63}$ Algunos autores consideran que la punción guiada por tomografía, ${ }^{64}$ resonancia o fluoroscopia mejora la precisión a la hora de localizar la espina isquiática y el nervio, ${ }^{6,10,13}$ pero no son fácilmente accesibles y exponen al operador a radiación ionizante. Actualmente, la ecografía tiene la misma tasa de éxito que las anteriores, evitando la exposición a la radiación y permitiendo la identificación tisular específica, así como la inserción de la aguja y de soluciones anestésicas en tiempo real. ${ }^{46,48,59,65}$

Las inyecciones se realizan con corticoides y anestésicos locales, y se aplican a nivel del espacio interligamentoso y en el canal de Alcock. ${ }^{9,66}$ La tasa de éxito de este procedimiento se 
Tabla 2 Cuestionario de evaluación para el síndrome de atrapamiento del nervio pudendo según Benson y Griffs

\begin{tabular}{|c|c|c|}
\hline \multicolumn{3}{|l|}{ ¿Desde hace cuánto tiempo tiene dolor? (días, meses o años) } \\
\hline \multicolumn{3}{|l|}{ ¿Hay algún evento que haya coincidido con el inicio del dolor? } \\
\hline \multicolumn{3}{|c|}{$\begin{array}{l}\text { ¿En una escala de dolor cuánto duele de } 0 \text { a } 10 \text { ? (Donde } 0 \text { es nada de dolor y } 10 \text { es el dolor } \\
\text { máximo que ha experimentado en su vida) }\end{array}$} \\
\hline \multicolumn{3}{|c|}{$\begin{array}{l}\text { ¿El dolor es: "quemante», «tirante», "aplastante», "presión», "penetrante», "cortante», otros? } \\
\text { (señalar con un círculo) }\end{array}$} \\
\hline Para las siguientes preguntas debe responder sí o no: & Sí & No \\
\hline \multicolumn{3}{|l|}{ ¿El dolor es mayor en un lado que en el otro? } \\
\hline \multicolumn{3}{|l|}{ ¿Tiene dolor en la región de las nalgas? } \\
\hline \multicolumn{3}{|l|}{ ¿Tiene dolor en la vagina o pene? } \\
\hline \multicolumn{3}{|l|}{ ¿Tiene dolor en los labios de la vulva o escroto? } \\
\hline \multicolumn{3}{|l|}{ ¿El dolor empeora al sentarse? } \\
\hline \multicolumn{3}{|c|}{ ¿Sentarse en el baño no es tan doloroso como sentarse en una superficie plana? } \\
\hline \multicolumn{3}{|l|}{ ¿El dolor es peor al estar de pie? } \\
\hline \multicolumn{3}{|l|}{ ¿El dolor es peor al acostarse? } \\
\hline \multicolumn{3}{|l|}{ ¿El dolor es peor al acostarse hacia un lado que al otro? } \\
\hline \multicolumn{3}{|l|}{ ¿Hay dolor con la defecación o movimiento intestinal? } \\
\hline \multicolumn{3}{|l|}{ ¿Hay dolor con la relación sexual? } \\
\hline \multicolumn{3}{|l|}{ ¿Hay dolor al orinar? } \\
\hline \multicolumn{3}{|l|}{ ¿Tiene problemas para controlar la vejiga -micción-? } \\
\hline \multicolumn{3}{|l|}{ ¿Tiene problemas para controlar el intestino -defecación-? } \\
\hline ¿Tiene problemas para controlar la expulsión de gases? & & \\
\hline
\end{tabular}

encuentra alrededor del 31-62\% en inyecciones únicas y aumenta en inyecciones repetidas con ciclos cada 6 semanas. ${ }^{5}$

El abordaje transglúteo se asocia con riesgo moderado de bloqueo concomitante de otras estructuras nerviosas. Fitchner Bendtsen et al. describieron la técnica de bloqueo en el interior del canal de Alcock guiado por ultrasonido, con menor riesgo de bloqueo del ciático. ${ }^{67}$

\section{Descompresión quirúrgica del nervio pudendo}

Se reserva para los pacientes con sospecha de atrapamiento que no han respondido adecuadamente al manejo conservador $^{3,37}$ y para aquellos cuya calidad de vida se ve deteriorada por la intensidad de los síntomas.
Diferentes autores han encontrado que el punto más frecuente de atrapamiento del nervio se localiza entre el ligamento sacroespinoso y el sacrotuberoso en cerca del $70 \%$ de los pacientes ya sea por anormalidades en los ligamentos (engrosamiento) o enfermedades que afecten puntualmente el nervio como fibrosis o procesos inflamatorios. ${ }^{36,68}$ Otras localizaciones menos frecuentes se presentan a nivel del canal de Alcock en el $20 \%$, en el diafragma urogenital, sobre el borde interno del proceso falciforme del ligamento sacrotuberoso o hacia la base del pene; ${ }^{6,8,12,43}$ en algunos reportes quirúrgicos se ha encontrado doble sitio de atrapamiento. $^{16}$

Conocer el lugar anatómico exacto donde se presenta la compresión permite que el cirujano planee el procedimiento ideal para obtener así mejores resultados.

Tabla 3 Criterios mayores y menores para el diagnóstico del síndrome de atrapamiento del nervio pudendo según Bautrant et al

\begin{tabular}{|l|l|}
\hline Criterios mayores & Criterios menores \\
\hline $\begin{array}{l}\text { Zona dolorosa en el trayecto del nervio pudendo: nervio } \\
\text { rectal inferior, perineal y dorsal del pene/clítoris }\end{array}$ & $\begin{array}{l}\text { Existencia de una posición dolorosa y/o antiálgica -empeora } \\
\text { sentado, mejora acostado }\end{array}$ \\
\hline Signo de Tinel positivo & Dolor de tipo neuropático \\
\hline $\begin{array}{l}\text { Infiltración anestésica exitosa con alivio del dolor durante } \\
\text { más de } 12 \text { h }\end{array}$ & $\begin{array}{l}\text { Existencia de un factor etiológico o desencadenante: } \\
\text { trauma, cirugía perineal, parto, deporte }\end{array}$ \\
\hline & $\begin{array}{l}\text { Ausencia de otra causa que explique el dolor en la zona } \\
\text { pélvica }\end{array}$ \\
\hline
\end{tabular}

Dos criterios mayores o un criterio mayor y dos menores se consideran diagnósticos para el síndrome de atrapamiento del nervio pudendo. 
Existen diferentes abordajes para realizar la descompresión quirúrgica - transglúteo, ${ }^{68}$ transperineal, transvaginal, isquiorrectal o laparoscópico ${ }^{69}$-, cada uno con tasas de éxito variables como mostramos en la - Tabla 4.

El abordaje perineal permite la liberación del nervio a nivel del canal de Alcock, pero no a nivel del área interligamentaria, que es el lugar más frecuente de compresión. ${ }^{36}$ El abordaje transglúteo se realiza disecando las fibras musculares hasta alcanzar el ligamento sacrotuberoso, liberando el nervio de las zonas de atrapamiento. Permite la visualización del trayecto extrapélvico del pudendo y facilita el acceso al espacio interligamentario y a la fosa isquiorrectal. ${ }^{16,68}$

Los resultados de la cirugía dependen en gran parte de una adecuada evaluación prequirúrgica, que permita diferenciar la compresión nerviosa de otras dolencias. ${ }^{70,71}$ Los resultados también dependen de la viabilidad de la fibra nerviosa. Se ha reportado que el $40 \%$ de los pacientes llevados a cirugía logra controlar el dolor; el 30\% solo manifiesta una mejoría parcial y en el $30 \%$ no hay ningún cambio; ${ }^{72}$ sin embargo, algunos autores han comparado la cirugía con el tratamiento conservador y el bloqueo pudendo, y han encontrado una superioridad en el tratamiento quirúrgico (60-71,4\% vs. $13,3 \%)^{71}$

La evaluación del resultado quirúrgico debe ser realizada a corto y a largo plazo, ya que la recuperación del nervio puede tardar más de un año.

Possover et al. describieron la neuronavegación laparoscópica con acceso retroperitoneal al plexo lumbosacro a través de una vía mínimamente invasiva. ${ }^{72} \mathrm{El}$ procedimiento se realiza identificando el espacio obturador a través de una disección parailíaca hacia el tronco lumbosacro. Luego, la disección se extiende hacia el aspecto más posterior de las raíces sacras. ${ }^{73}$ Para este tipo de cirugías se recomienda el uso de cámaras de alta resolución y un neuroestimulador intraoperatorio durante la disección de los nervios. ${ }^{74}$ Se utiliza un puerto umbilical para la cámara y 3 puertos adicionales, localizados en el hemiabdomen inferior - uno en la línea media y los otros laterales a los vasos epigástricos de cada lado $^{26}$-. Para la exposición de las raíces sacras de S1-S4, la disección inicia a nivel del espacio pararrectal medial al uréter y continua caudal, hasta el hueso coccígeo. La exposición del nervio pudendo se logra iniciando la disección a nivel de la fosa lumbosacra, lateral a los vasos ilíacos externos y siguiendo el músculo obturador interno. ${ }^{26}$ En la mayoría de los casos se logra la identificación topográfica de la lesión, más no la del sitio de atrapamiento.

La implantación de un neuromodulador por vía abierta o laparoscópica se ha utilizado, con resultados prometedores que deben continuar evaluándose en los casos de falla terapéutica. ${ }^{72,75,76}$ El procedimiento de implantación laparoscópica de neuroprótesis (LION procedure) está indicado cuando no hay una causa clara de los síntomas neurológicos, cuando hay sospecha de lesión de las fibras nerviosas, o cuando los hallazgos de la laparoscopia no son suficientes para explicar los síntomas del paciente. ${ }^{26}$ Se realiza la implantación de un electrodo multicanal en contacto directo con el nervio pudendo. ${ }^{53}$

La técnica de Estambul descrita por Sancak et al. implica la liberación del nervio en el sitio de atrapamiento por vía laparoscópica y la creación de un flap de omento que rodea al nervio liberado, para evitar la formación de fibrosis alrededor. ${ }^{37}$

Las complicaciones quirúrgicas son poco frecuentes e incluyen: infección de vías urinarias, abscesos pélvicos, lesión de arteria pudenda o formaciones aneurismáticas, hematomas, dolor persistente o aparición de dolor neuropático transitorio en la región glútea. ${ }^{8,12}$

Tabla 4 Abordajes quirúrgicos, EVA

\begin{tabular}{|c|c|c|c|c|c|c|}
\hline Autor & Año & $\begin{array}{l}\text { N. }{ }^{\circ} \text { de pa- } \\
\text { cientes }\end{array}$ & Edad & Vía quirúrgica & $\begin{array}{l}\text { Mejoría } \\
>50 \% \text { por } \\
\text { EVA }\end{array}$ & $\begin{array}{l}\text { Promedio de } \\
\text { seguimiento } \\
\text { (meses) }\end{array}$ \\
\hline Beco & 2004 & 74 & $56,1(22-77)$ & Isquiorrectal & $77,7 \%$ & 22,2 \\
\hline Bautrant & 2003 & 104 & $52(19-81)$ & $\begin{array}{l}\text { Transvaginal/ } \\
\text { Isquiorrectal }\end{array}$ & $86 \%$ & 12 \\
\hline Benson & 2005 & 10 & $30-71$ & Transglúteo & $60 \%$ & No hay datos \\
\hline Mauillon & 1999 & 12 & $63,2(50-76)$ & Transglúteo & $29,1 \%$ & $63,2(50-76)$ \\
\hline Filler & 2009 & 147 & No hay datos & Transglúteo & $70 \%$ & No hay datos \\
\hline Dellon & 2015 & 55 & $39,5(31-75)$ & $\begin{array}{l}\text { Transvaginal/ } \\
\text { Transglúteo }\end{array}$ & $49 \%$ & 36 \\
\hline Shafik & 1998 & 11 & $38,4(22-53)$ & $\begin{array}{l}\text { Isquiorrectal/ } \\
\text { Transperineal }\end{array}$ & $81,8 \%$ & $22,6(12-38)$ \\
\hline Possover & 2007 & 7 & $49,6(30-58)$ & $\begin{array}{l}\text { LION } \\
\text { procedure }\end{array}$ & $100 \%$ & $10,7(5-13)$ \\
\hline Possover & 2009 & 127 & No hay datos & Laparoscópica & $62 \%$ & $17(3-39)$ \\
\hline Erdogru & 2013 & 27 & $43,9(25-58)$ & $\begin{array}{l}\text { Laparoscópica } \\
\text { con flap de } \\
\text { omento }\end{array}$ & $100 \%$ & $6,8(1-15)$ \\
\hline
\end{tabular}

EVA: escala visual análoga. 


\section{Conclusiones}

El dolor pélvico por compresión del nervio pudendo es un síndrome poco conocido, que se debe tener en cuenta en los pacientes que consultan por dolor en pelvis o periné. El mayor reto en este tipo de enfermedades está en el diagnóstico oportuno. Ello permitirá realizar un abordaje multidisciplinario secuencial en busca de mejores opciones de tratamiento para estos pacientes que presentan un importante compromiso de su calidad de vida.

\section{Financiación}

Las autoras declaran no haber recibido ningún tipo de apoyo financiero para la realización del presente trabajo.

\section{Conflicto de intereses}

Las autoras declaran no tener ningún conflicto de intereses relacionados con la publicación del presente artículo.

\section{Agradecimientos}

Al Dr. Alfredo Ortiz MD Urólogo y a la Dra. Natalia Jaramillo MD Radióloga, por sus aportes y sugerencias en la realización del presente documento.

\section{Bibliografía}

1 Amarenco G, Lanoe Y, Perrigot M, Goudal H, et al. A new canal syndrome: Compression of the pudendal nerve in Alcock's canal or perinal paralysis of cyclists. Press Med 1987;16:399

2 Itza Santos F, Salinas J, Zarza D, Gómez Sancha F, Allona Almagro A. Update in pudendal nerve entrapment syndrome: an approach anatomic-surgical, diagnostic and therapeutic. Actas Urol Esp 2010;34(06):500-9

3 Khoder W, Hale D. Pudendal neuralgia. Obstet Gynecol Clin North Am 2014;41:443-452

4 Maldonado PA, Chin K, Garcia AA, Corton MM. Anatomic variations of pudendal nerve within pelvis and pudendal canal: Clinical applications. Am J Obstet Gynecol 2015;213:727e1-727e6

5 Benson JT, Griffis K. Pudendal neuralgia, a severe pain syndrome. Am J Obstet Gynecol 2005;192:1663-1668

6 Labat J-J, Riant T, Robert R, Amarenco G, Lefaucheur JP, Rigaud J. Diagnostic criteria for pudendal neuralgia by pudendal nerve entrapment (Nantes criteria). Neurourol Urodyn 2008;27:306-310

7 Hibner M, Desai N, Robertson LJ, Nour M. Pudendal neuralgia. J Minim Invasive Gynecol 2010;17:148-153

8 LabatJ. Orpha.net [Internet]. 2014 [citado 1 Abr 2014 1]. Disponible en: http://www.orpha.net/consor/cgi-bin/Disease_Search.php? lng=EN\&data_id=10804\&Disease_Disease_Search_diseaseGroup= Pudendal-neuralgia\&Disease_Disease_Search_diseaseType=Pat\& Disease(s)/groupofdiseases=Pudendal-neuralgia\&title=Pudendalneuralgia\&search $=$ Disea

9 Lema CR, Ricci AP. Atrapamiento del nervio oudendo: Un síndrome por conocer. Rev Chil Obstet Ginecol 2006;71:207-215

10 Colebunders B, Matthew MK, Broer N, Persing JA, Dellon AL. Benjamin Alcock and the pudendal canal. J Reconstr Microsurg 2011;27:349-353

11 Hruby S, Ebmer J, Dellon AL, Aszmann OC. Anatomy of pudendal nerve at urogenital diaphragm - New critical site for nerve entrapment. Urology 2005;66:949-952

12 Robert R, Prat-Pradal D, Labat JJ, Bensignor M, Raoul S, Rebai R, et al. Anatomic basis of chronic perineal pain: Role of the pudendal nerve. Surg Radiol Anat 1998;20:93-98
13 Mahakkanukrauh P, Surin P, Vaidhayakarn P. Anatomical study of the pudendal nerve adjacent to the sacrospinous ligament. Clin Anat 2005; 18:200-205

14 Gabrielli C, Olave E. Aspectos anatómicos y topográficos del nervio pudendo en la región glútea. Int J Morphol 2011;29:168-173

15 Furtmüller GJ, McKenna CA, Ebmer J, Dellon AL. Pudendal nerve 3dimensional illustration gives insight into surgical approaches. Ann Plast Surg 2014;73:670-678

16 Ploteau S, Perrouin-Verbe M-A, Labat J, Riant T, Levesque A, Robert R. Anatomical variants of the pudendal nerve observed during a transgluteal surgical approach in a population of patients with pudendal neuralgia. Pain Physician 2017;20:137-144

17 Ogiwara $\mathrm{H}$, Morota N. Pudendal afferents mapping in posterior sacral rhizotomies. Neurosurgery 2014;74:171-175

18 Shafik A, El-Sherif M, Youssef A, Olfat ES. Surgical anatomy of the pudendal nerve and its clinical implications. Clin Anat 1995;8:110-115

19 Hwang K, Nam YS, Kim DJ, Han SH, Hwang SH. Posterior cutaneous nerve of the thigh relating to the restoration of the gluteal fold. Ann Plast Surg 2008;60:357-361

20 Sedy J, Nanka O, Spaskova J, Jarolim L. Clinical implications of a close vicinity of nervus dorsalis penis/clitoridis and os pubis. J Sex Med 2008;5:1572-1581

21 Venegas-Ocampo P, Castillo-De Lira H, Robles-Scott M, LandaSoler M, López-Mariscal M, Mendoza-Peña FV-M.R. Comparación de la efectividad del bloqueo perineal de los nervios pudendos como método anestésico en la biopsia transrectal de próstata guiada por ultrasonido. Rev Mex Urol 2010;70:164-170

22 Réding CM, Ramírez P, Villagómez Z, Borbolla IR. Bloqueo de Nervios Pudendos en Obstetricia. In: Trabajo presentado en el IX Congreso Mexicano de Anestesiología; 1962 Nov; Mexico DF; 1962. p. $107-11$

23 Van der Walt S, Oettlé AC, van Wijk FJ. The pudendal nerve and its branches in relation to Richter's procedure. Gynecol Obstet Invest 2016;81:275-279

24 O'Bichere A, Green C, Phillips RK. New, simple approach for maximal pudendal nerve exposure: anomalies and prospects for functional reconstruction. Dis Colon Rectum 2000;43:956-960

25 Bondar A, Egan M, Jochum D, Amarenco G, Bouaziz H. Case report: Pudendal nerve injury after a sciatic nerve block by the posterior approach. Anesth Analg 2010;111:573-575

26 Possover M, Lemos N. Risks, symptoms, and management of pelvic nerve damage secondary to surgery for pelvic organ prolapse: A report of 95 cases. Int Urogynecol 2011;22:1485-1490

27 Itza F, Zarza D, Gomez F. Patologías más frecuentes que cursan con dolor pélvico crónico: actualización clínica y diagnostica. Urodinamica Apl 2010;23:6-22

28 Reitz A, Schmid DM, Curt A, Knapp PA, Schurch B. Afferent fibers of the pudendal nerve modulate sympathetic neurons controlling the bladder neck. Neurourol Urodyn 2003;22:597-601

29 Lemos N, Possover M. Laparoscopic approach to intrapelvic nerve entrapments. J Hip Preserv Surg 2015;2:92-98

30 Marchand F, Perretti M, McMahon SB. Role of the immune system in chronic pain. Nat Rev Neurosci [Internet] 6:521-5322005 Disponible en: http://www.ncbi.nlm.nih.gov/pubmed/15995723

31 Possover M, Forman A. Pelvic neuralgias by neuro-vascular entrapment: Anatomical findings in a series of 97 consecutive patients treated by laparoscopic nerve decompression. Pain Physician 2015;18:1139-1144

32 Burnett MG, Zager EL. Pathophysiology of peripheral nerve injury: A brief review. Neurosurg Focus 2004;16:E1E

33 Itza Santos F, Salinas J, Zarza D, Gómez Sancha F, Allona Almagro A. Update in pudendal nerve entrapment syndrome: An approach anatomic-surgical, diagnostic and therapeutic. Actas Urol Esp 2010;34:500-509

34 Elahi F, Callahan D, Greenlee J, Dann TL. Pudendal entrapment neuropathy: A rare complication of pelvic radiation therapy. Pain Physician 2013;16:E793-E797 
35 Lim JF, Tjandra JJ, Hiscock R, Chao MW.T, Gibbs P. Preoperative chemoradiation for rectal cancer causes prolonged pudendal nerve terminal motor latency. Dis Colon Rectum 2006;49:12-19

36 Bautrant E, de Bisschop E, Vaini-Elies V, Massonnat J, Aleman I, Buntinx J, et al. Modern algorithm for treating pudendal neuralgia: 212 cases and 104 decompressions. J Gynecol Obstet Biol Reprod 2003;32(8 Pt 1): 705-712

37 Sancak EB, Avci E, Erdogru T. Pudendal neuralgia after pelvic surgery using mesh: Case reports and laparoscopic pudendal nerve decompression. Int J Urol 2016;23:797-800

38 Chung CP, Kuehl TJ, Larsen WI, Yandell PM, Shull BL. Recognition and management of nerve entrapment pain after uterosacral ligament suspension. Obstet Gynecol 120(2 Pt 1):2012:292-295

39 Noda Miranda JJ, Díaz Noda V. Síndrome de atrapamiento del nervio pudendo. Rev Cuba Obstet y Ginecol 2013;39:167-175

40 Beco J, Liege B. Pudendal neuropathy: Current views. International Continence Society - 41st anual meeting, Glasgow UK, 2011

41 Ploteau S, Cardaillac C, Riant T, Labat J. Pudendal neuralgia due to pudendal nerve entrapment: Warning signs observed in two cases and review of the literature. Pain Physician 2016;19:449-454

42 Mollo M, Bautrant E, Rossi-Seignert AK, Collet S, Boyer R, ThiersBautrant D. Evaluation of diagnostic accuracy of Colour Duplex Scanning, compared to electroneuromyography, diagnostic score and surgical outcomes, in pudendal neuralgia by entrapment: A prospective study on 96 patients. Pain 2009;142:159-163

43 Lefaucheur JP, Labat JJ, Amarenco G, Herbaut AG, Prat-Pradal D, Benaim J, et al. What is the place of electroneuromyographic studies in the diagnosis and management of pudendal neuralgia related to entrapment syndrome? Neurophysiol Clin 2007;37:223-228

44 Antolak S, Antolak C, Lendway L. Measuring the quality of pudendal nerve perineural injections. Pain Med (United States) 2016;19:299-306

45 Amarenco G, Kerdraon J. Pudendal nerve terminal sensitive latency: Technique and normal values. J Urol 1999;161:103-106

46 Roche B, Robert-Yap J, Skala K. Pudendal nerve compression syndrome. Soc Ital di Chir Color 2009;20:172-179

47 Chhabra A, Andreisek G, Soldatos T, Wang KC, Flammang AJ, Belzberg AJ, et al. MR neurography: Past, present, and future. Am J Roentgenol 2011;197:583-591

48 Filler A. Magnetic resonance neurography and diffusion tensor imaging: Origins, history, and clinical impact of the first 50,000 cases with an assessment of efficacy and utility in a prospective 5,000 patient study group. Neurosurgery 2009;65:A29-A43

49 Filler AG. IKNOWLEDGE - Imaging for peripheral nerve disorders [Internet]. 2015 [citado 13 Mar 2015]. Chapter 196. Disponible en: http://clinicalgate.com/imaging-for-peripheral-nerve-disorders/

50 Fillier A. Diagnosis and treatment of pudendal nerve entrapment syndrome subtypes: Imaging, injections, and minimal access surgery. Neurosurg Focus 2009;26:E9

51 Possover M, Schneider T, Henle KP. Laparoscopic therapy for endometriosis and vascular entrapment of sacral plexus. Fertil Steril 2011;95:756-758

52 Moser T, Scheiber-Nogueira MC, Nogueira TS, Doll A, Jahn C, Beaujeux R. Neurological picture. Pudendal nerve compression by pelvic varices: Successful treatment with transcatheter ovarian vein embolisation. J Neurol Neurosurg Psychiatry 2006;77:88

53 Boelens OB, van Assen T, Houterman S, Scheltinga MR, Roumen RM. A double-blind, randomized, controlled trial on surgery for chronic abdominal pain due to anterior cutaneous nerve entrapment syndrome. Ann Surg 2013;257:845-849

54 Amarenco G, Raibaut P, Ismael SS, Rene-Corail P, Haab F. Evidence of occult dysautonomia in Fowler's syndrome: Alteration of cardiovascular autonomic function tests in female patients presenting with urinary retention. BJU Int 2006;97:288-291

55 Possover M, Forman A. Voiding dysfunction associated with pudendal nerve entrapment. Curr Bladder Dysfunct Rep 2012;7:281-285

56 Stav K, Dwyer PL, Roberts L. Pudendal neuralgia. Fact or fiction? Obstet Gynecol Surv 2009;64:190-199
57 Rhame EE, Levey KA, Gharibo CG. Successful treatment of refractory pudendal neuralgia with pulsed radiofrequency. Pain Physician 2009;12:633-638

58 Pereira A, Pérez-Medina T, Rodríguez-Tapia A, Rutherford S, Millan I, Iglesias E, et al. Chronic perineal pain: analyses of prognostic factors in pudendal neuralgia. Clin J Pain 2014;30:577-582

59 Bellingham GA, Bhatia A, Chan C-W, Peng PW. Randomized controlled trial comparing pudendal nerve block under ultrasound and fluoroscopic guidance. Reg Anesth Pain Med 2012;37:262-266

60 Gajraj NM. Botulinum toxin A injection of the obturator internus muscle for chronic perineal pain. J Pain 2005;6:333-337

61 Levesque A, Riant T, Labat J-J, Ploteau S. Use of high-concentration capsaicin patch for the treatment of pelvic pain: Observational study of 60 inpatients. Pain Physician 2017;20:161-168

62 Calabrò RS, Gervasi G, Marino S, Mondo PN. Case reports. Misdiagnosed chronic pelvic pain: Pudendal. Pain Med 2010: 781-784

63 Ricci P, Lema R, Sola V, Wash A, Pardo J. Infiltración del nervio pudendo guiada por tomografía axial computada, por vía transglutea: terapia frente al dolor ocasionado por neuralgia del nervio pudendo. Rev Chil Obstet Ginecol 2009;74:94-101

64 Calvillo O, Skaribas IM, Rockett C. Computed tomography-guided pudendal nerve block. A new diagnostic approach to long-term anoperineal pain: A report of two cases. Reg Anesth Pain Med 2000;25:420-423

65 Bhatia A, Brull R. Is ultrasound guidance advantageous for interventional pain management? A systematic review of chronic pain outcomes. Anesth Analg 2013;117:236-251

66 Asensio JM. Bloqueos ecodirigidos toracico lumbar y sacro en una unidad de tratamiento del dolor. Sociedad Europea de Anestesia Regional y Tratamiento del Dolor 2009:571-588

67 Bendtsen TF, Parras T, Moriggl B, Chan V, Lundby L, Buntzen S, et al. Ultrasound-guided pudendal nerve block at the entrance of the pudendal (Alcock) canal: Description of anatomy and clinical technique. Reg Anesth Pain Med 2016;41:140-145

68 Ploteau S, Perrouin M-A, Labat J, Riant T, Levesque A, Robert R. Anatomical variants of the pudendal nerve observed during a transgluteal surgical approach in a population of patients with pudendal neuralgia. Pain Physician J 2017;20:137-144

69 Shafik A. Pudendal canal syndrome as a cause of vulvodynia and its treatment by pudendal nerve decompression. Eur J Obstet Gynecol Reprod Biol 1998;80:215-220

70 Dellon AL, Coady D, Harris D. Pelvic pain of pudendal nerve origin: Surgical outcomes and learning curve lessons. J Reconstr Microsurg 2015;31:283-290

71 Robert R, Labat JJ, Bensignor M, Glemain P, Deschamps C, Raoul S, et al. Decompression and transposition of the pudendal nerve in pudendal neuralgia: A randomized controlled trial and long-term evaluation. Eur Urol 2005;47:403-408

72 Possover M, Baekelandt J, Chiantera V. The laparoscopic approach to control intractable pelvic neuralgia: From laparoscopic pelvic neurosurgery to the LION procedure. Clin J Pain 2007;23:821-825

73 Lemos N, Kamergorodsky G, Ploger C, Castro R, Schor E, Girão M. Sacral nerve infiltrative endometriosis presenting as perimenstrual right-sided sciatica and bladder atonia: Case report and description of surgical technique. J Minim Invasive Gynecol 2012;19:396-400

74 Lemos N, Souza C, Marques RM, Kamergorodsky G, Schor E, Girão MJ.B.C. Laparoscopic anatomy of the autonomic nerves of the pelvis and the concept of nerve-sparing surgery by direct visualization of autonomic nerve bundles. Fertil Steril 2015;104:11-12

75 Possover M. Use of the LION procedure on the sensitive branches of the lumbar plexus for the treatment of intractable postherniorrhaphy neuropathic inguinodynia. Hernia 2013;17:333-337

76 Valovska A, Peccora CD, Philip CN, Kaye AD, Urman RD. Sacral neuromodulation as a treatment for pudendal neuralgia. Pain Physician 2014;17:645-650 